\title{
Highly efficient conversion of aromatic acylals to 3, 4- dihydropyrimidinones: a new protocol for the Biginelli reaction
}

\author{
Hojatollah Khabazzadeh, Kazem Saidi*, and Hassan Sheibani \\ Department of Chemistry, Shahid Bahonar University of Kerman \\ 76169, Kerman, Iran \\ E-mail: saidik@mail.uk.ac.ir
}

\begin{abstract}
A novel version of the Biginelli reaction using an acylal as a masked carbonyl functionality together with ethyl acetoacetate and urea or thiourea to give dihydropyrimidinones is reported. The reaction is catalyzed by 12-tungstophosphoric acid (PW), 12-molybdophosphoric acid (PMo) or zinc chloride and performed in one-pot under solvent-free conditions.
\end{abstract}

Keywords: Dihydropyrimidinone, acylal, solvent-free, Biginelli reaction, heteropoly acid, zinc chloride

\section{Introduction}

In recent years, use of eco-friendly applicable industrial and green catalysts has been of interest. Thus, green chemistry has been defined as a set of principles that reduces or eliminates the use or generation of hazardous substances throughout the entire life of chemical materials. ${ }^{1}$ Along this line, using heteropoly acids (HPAs) has found more attention. ${ }^{2-4}$ HPAs have several advantages as catalysts which make them economically and environmentally feasible. They are stronger acids than homogeneous acid catalysts such as sulfuric acid or ion exchange resins. The use of HPAs as catalyst is important in the development of clean technologies, since it avoids the drawbacks of environmental pollution and prevents corrosion of the conventional technologies.

Multicomponent reactions (MCRs) are of increasing importance in organic and medicinal chemistry. ${ }^{5}$ In a time when a premium is put on speed, diversity and efficiency in the drug discovery process, ${ }^{6}$ MCR strategies offer significant advantages over conventional linear-type syntheses. In such reactions, three or more reactants come together in a single reaction vessel to form new products that contain portions of all the components. The search and discovery for new MCRs on one hand, and the full exploitation of the already known multicomponent reactions on the other hand, is therefore of considerable current interest. One such MCR that belongs to the latter category is the Biginelli; dihydropyrimidinone (DHPM) synthesis. 
Dihydropyrimidinones (DHPMs) and their derivatives have attracted considerable interest because of their pharmaceutical and therapeutic properties, such as antibacterial, antiviral, antitumour and anti-inflammatory activities. ${ }^{7-9}$ The simple and direct method for the synthesis of dihydropyrimidinones was first reported by Biginelli in 1893, involving a one-pot condensation of an aldehyde, $\beta$-ketoester and urea under strongly acidic conditions. ${ }^{10}$ However, it suffers from low yields $(20-50 \%)$ of products. Therefore, several improved methodologies mainly using Lewis acids, ${ }^{11,}{ }^{12}$ triflates, ${ }^{13}$ microwave-assisted methodologies ${ }^{14}$ and ultrasonic mediated methods ${ }^{15}$ have been reported in the literature. However, in spite of their potential utility many of the existing methods suffers from the drawbacks such as the use of strong acidic conditions, longer reaction times, tedious workup, environmental disposal problems and lower yields of the products, leaving scope for further development of an efficient and versatile method for Biginelli reaction.

In over 114 years of study of the Biginelli reaction, only minor structural variations in its three building blocks have been reported, ${ }^{16}$ apart from a recently reported major structural variation where the urea component was replaced by a guanidine system. ${ }^{17}$ However, to the best of our knowledge, there has been no structural variation in the carbonyl building block; this could result in a novel version of the Biginelli reaction for the synthesis of DHPMs.

Acylals (1,1-diacetates) have been recognized as an important protecting group alternative to acetals because of their stability under various reaction conditions and their easy conversion into the parent aldehydes. ${ }^{18}$ Being a masked aldehydic functionality, acylals have been used as a valuable intermediate in various organic syntheses. ${ }^{19-21}$ Acylals have been used as precursors of 1-acetoxydienes, 2, 2-dichlorovinylacetates for Diels-Alder reactions. ${ }^{22}$ Herein, we report the first example of the Biginelli reaction employing acylals (Scheme 1).

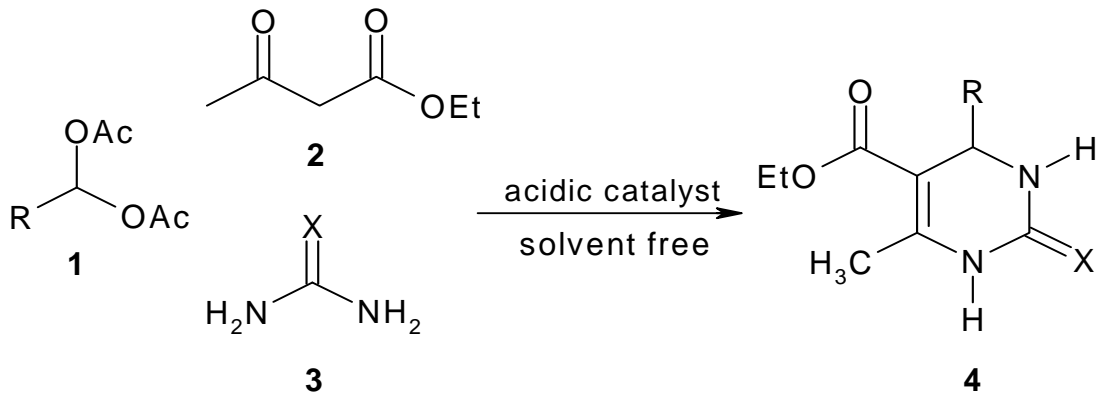

\section{Scheme 1}

\section{Results and Discussion}

The three component Biginelli condensation by examining conditions for the reaction involving acylal of benzaldehyde, urea and ethyl acetoacetate as a model reaction to synthesis DHPM 4a $(\mathrm{R}=\mathrm{Ph}, \mathrm{X}=\mathrm{O})$ were investigated. To establish the optimal conditions, a set of experiments 
varying the amount of the catalysts were carried out. The best conditions to prepare the DHPMs were achieved when $10 \mathrm{~mol} \%, 20 \mathrm{~mol} \%$, and $80 \mathrm{~mol} \%$ of PW, PMo, and $\mathrm{ZnCl}_{2}$ were used, respectively. The results are summarized in table 1.

Table 1. Effect of different catalysts on the condensation reaction of acylal of benzaldehyde, ethyl acetoacetate and urea

\begin{tabular}{clcc}
\hline Entry & Catalyst (mol \%) & Time (min) & Yield (\%) \\
\hline 1 & $\mathrm{PW} \mathrm{(5)}$ & 50 & 60 \\
2 & $\mathrm{PW} \mathrm{(10)}$ & 20 & 80 \\
3 & $\mathrm{PMo} \mathrm{(5)}$ & 60 & 50 \\
4 & $\mathrm{PMo} \mathrm{(10)}$ & 60 & 58 \\
5 & $\mathrm{PMo} \mathrm{(15)}$ & 40 & 70 \\
6 & $\mathrm{PMo} \mathrm{(20)}$ & 20 & 75 \\
7 & $\mathrm{ZnCl}_{2}(10)$ & 90 & 30 \\
8 & $\mathrm{ZnCl}_{2}(20)$ & 60 & 45 \\
9 & $\mathrm{ZnCl}_{2}(40)$ & 60 & 56 \\
10 & $\mathrm{ZnCl}_{2}(50)$ & 40 & 60 \\
11 & $\mathrm{ZnCl}_{2}(80)$ & 40 & 70 \\
\hline
\end{tabular}

These optimum conditions were applied to a series of substituted aromatic acylals. The results are shown in Table 2. The reaction of aromatic acylals containing electron-donating substituents accelerated the reaction and giving high yields in short reaction times. The presence of electron-withdrawing groups on aromatic acylals retards the reaction and causes to increase the reaction time. This observation shows that this reaction is strongly affected by the electronic factors. Another factor that affects the reaction time and yield is steric hinderance. Acylals containing ortho substituents such as $\mathbf{4 c}$ and $\mathbf{4 h}$ react more slowly. 
Table 2. Biginelli reaction using acylals, ethyl acetoacetate and urea/thiourea

\begin{tabular}{|c|c|c|c|c|c|c|c|c|}
\hline \multirow{2}{*}{ Entry } & \multirow{2}{*}{ Product } & \multirow{2}{*}{$\mathrm{R}$} & \multirow{2}{*}{$\mathrm{X}$} & \multicolumn{2}{|c|}{$\operatorname{Mp}\left({ }^{\circ} \mathrm{C}\right)$} & \multicolumn{3}{|c|}{ Yield(\%)/time(min) } \\
\hline & & & & observed & reported $^{\text {ref }}$ & $\mathrm{PW}^{\mathrm{a}}$ & $\mathrm{PMo}^{\mathrm{b}}$ & $\mathrm{ZnCl}_{2}{ }^{\mathrm{c}}$ \\
\hline 1 & $4 a$ & $\mathrm{Ph}$ & $\mathrm{O}$ & 199-202 & $198-200^{23}$ & $80 / 20$ & $75 / 20$ & $80 / 40$ \\
\hline 2 & $4 b$ & 4- $\mathrm{ClC}_{6} \mathrm{H}_{4}$ & $\mathrm{O}$ & $210-212$ & $211-213^{23}$ & $85 / 25$ & $80 / 35$ & $85 / 40$ \\
\hline 3 & $4 c$ & $2-\mathrm{ClC}_{6} \mathrm{H}_{4}$ & $\mathrm{O}$ & $212-215$ & $216-218^{24}$ & $81 / 50$ & $70 / 60$ & $70 / 70$ \\
\hline 4 & $4 d$ & $3-\mathrm{O}_{2} \mathrm{NC}_{6} \mathrm{H}_{4}$ & $\mathrm{O}$ & $226-228$ & $229-230^{25}$ & $70 / 55$ & $60 / 60$ & $60 / 70$ \\
\hline 5 & $4 e$ & $4-\mathrm{O}_{2} \mathrm{NC}_{6} \mathrm{H}_{4}$ & $\mathrm{O}$ & $202-205$ & $205-207^{24}$ & $66 / 60$ & $60 / 70$ & $65 / 70$ \\
\hline 6 & 4f & 1-Naphthyl & $\mathrm{O}$ & $253-255$ & $256-258^{27}$ & $85 / 10$ & $83 / 15$ & $75 / 15$ \\
\hline 7 & $4 g$ & $4-\mathrm{CH}_{3} \mathrm{C}_{6} \mathrm{H}_{4}$ & $\mathrm{O}$ & $210-213$ & $213-216^{23}$ & $86 / 20$ & $85 / 25$ & $85 / 30$ \\
\hline 8 & $4 h$ & $2-\mathrm{CH}_{3} \mathrm{C}_{6} \mathrm{H}_{4}$ & $\mathrm{O}$ & 207-209 & $208-210^{28}$ & $80 / 40$ & $74 / 50$ & $75 / 50$ \\
\hline 9 & $4 i$ & 4- $\mathrm{CH}_{3} \mathrm{OC}_{6} \mathrm{H}_{4}$ & $\mathrm{O}$ & $200-202$ & $203-205^{23}$ & $85 / 20$ & $85 / 30$ & $80 / 30$ \\
\hline 10 & $4 j$ & $2,4-(\mathrm{Cl})_{2} \mathrm{C}_{6} \mathrm{H}_{3}$ & $\mathrm{O}$ & $243-245$ & $246-248^{23}$ & $83 / 50$ & $80 / 60$ & $80 / 65$ \\
\hline 11 & $4 k$ & $\mathrm{Ph}$ & $\mathrm{S}$ & $198-200$ & $202-204^{23}$ & $86 / 50$ & $80 / 60$ & $70 / 60$ \\
\hline 12 & 41 & $4-\mathrm{CH}_{3} \mathrm{C}_{6} \mathrm{H}_{4}$ & $\mathrm{~S}$ & $186-188$ & $189-192^{26}$ & $85 / 50$ & $78 / 50$ & $74 / 55$ \\
\hline 13 & $4 m$ & $4-\mathrm{ClC}_{6} \mathrm{H}_{4}$ & $\mathrm{~S}$ & $180-182$ & $184-185^{23}$ & $76 / 60$ & $70 / 65$ & $73 / 65$ \\
\hline
\end{tabular}

${ }^{a}$ Reaction conditions: acylal $(1 \mathrm{mmol})$, ethyl acetoacetate $(1 \mathrm{mmol})$, urea/thiourea $(1.2 \mathrm{mmol})$, PW (0.1mmol)

${ }^{\mathrm{b}}$ Reaction conditions: acylal $(1 \mathrm{mmol})$, ethyl acetoacetate $(1 \mathrm{mmol})$, urea/thiourea $(1.2 \mathrm{mmol})$, PMo (0.2mmol)

${ }^{\mathrm{c}}$ Reaction conditions: acylal $(1 \mathrm{mmol})$, ethyl acetoacetate $(1 \mathrm{mmol})$, urea/thiourea $(1.2 \mathrm{mmol})$, $\mathrm{ZnCl}_{2}(0.8 \mathrm{mmol})$

This reaction may proceed via acyl imine intermediate 5 , formed by the reaction of the acylal and urea. Subsequent addition of $\beta$-ketoester enole to the acylimine, followed by cyclization and dehydration, afforded the corresponding dihydropyrimidinones (Scheme 2). ${ }^{29}$ 


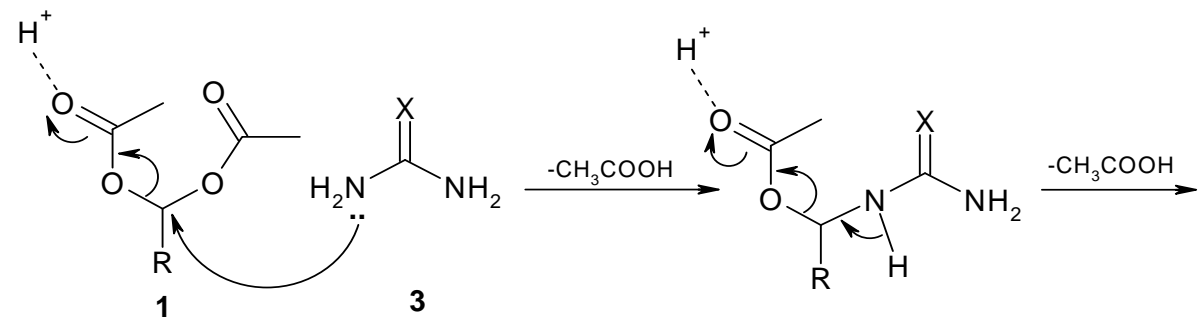<smiles>[R]C=C(C)[NH2+]C(N)=[Y][CH]C(=O)OCC</smiles>

\section{Scheme 2}

To show the merit of the present work results were compared with the reported results in the literature in table 3.

Table 3. Comparison of present work with literature results

\begin{tabular}{|c|c|c|c|c|c|}
\hline Entry & Substrate & Catalyst & Condition & Time & Yield $(\%)^{\text {ref }}$ \\
\hline \multirow{3}{*}{1} & & $\mathrm{H}_{3} \mathrm{PW}_{12} \mathrm{O}_{40}(2 \mathrm{~mol} \%)$ & Acetic acid, reflux & $6 \mathrm{~h}$ & $75^{30}$ \\
\hline & & $\mathrm{H}_{3} \mathrm{PMo}_{12} \mathrm{O}_{40}(2 \mathrm{~mol} \%)$ & Acetic acid, reflux & $4 \mathrm{~h}$ & $70^{31}$ \\
\hline & & Fluorapatit- $\mathrm{ZnCl}_{2}(0.2 \mathrm{~g})$ & Toluene, reflux & $72 \mathrm{~h}$ & $78^{32}$ \\
\hline \multirow{3}{*}{2} & & $\mathrm{H}_{3} \mathrm{PW}_{12} \mathrm{O}_{40}(10 \mathrm{~mol} \%)$ & Solvent-free, $100^{\circ} \mathrm{C}$ & $20 \mathrm{~min}$ & 85 \\
\hline & & $\mathrm{H}_{3} \mathrm{PMo}_{12} \mathrm{O}_{40}(20 \mathrm{~mol} \%)$ & Solvent-free, $100^{\circ} \mathrm{C}$ & $30 \mathrm{~min}$ & 85 \\
\hline & & $\mathrm{ZnCl}_{2}(80 \mathrm{~mol} \%)$ & Solvent-free, $100^{\circ} \mathrm{C}$ & $30 \mathrm{~min}$ & 80 \\
\hline \multirow{3}{*}{3} & & $\mathrm{H}_{3} \mathrm{PW}_{12} \mathrm{O}_{40}(2 \mathrm{~mol} \%)$ & Acetic acid, reflux & $6 \mathrm{~h}$ & $70^{30}$ \\
\hline & & $\mathrm{H}_{3} \mathrm{PMo}_{12} \mathrm{O}_{40}(2 \mathrm{~mol} \%)$ & Acetic acid, reflux & $6 \mathrm{~h}$ & $80^{31}$ \\
\hline & & Fluorapatit- $\mathrm{ZnCl}_{2}(0.2 \mathrm{~g})$ & Toluene, reflux & $72 \mathrm{~h}$ & $82^{32}$ \\
\hline \multirow{3}{*}{4} & & $\mathrm{H}_{3} \mathrm{PW}_{12} \mathrm{O}_{40}(10 \mathrm{~mol} \%)$ & Solvent-free, $100^{\circ} \mathrm{C}$ & $25 \mathrm{~min}$ & 85 \\
\hline & & $\mathrm{H}_{3} \mathrm{PMo}_{12} \mathrm{O}_{40}(20 \mathrm{~mol} \%)$ & Solvent-free, $100^{\circ} \mathrm{C}$ & $35 \mathrm{~min}$ & 80 \\
\hline & & $\mathrm{ZnCl}_{2}(80 \mathrm{~mol} \%)$ & Solvent-free, $100^{\circ} \mathrm{C}$ & $40 \mathrm{~min}$ & 85 \\
\hline
\end{tabular}




\section{Conclusions}

In conclusion, we have demonstrated that readily available acylals in a one pot synthesis undergo facile reaction with urea/thiourea and ethyl acetoacetate to give dihydropyrimidinones under solvent free conditions. Acylals are persistent to the oxidation so they can be easily used with Lewis acids that are capable to oxidize aldehydes. It is pertinent to note that lower amounts of water is produced in this reaction, therefore those catalysts which are susceptible to water can preserve their activity until the final step of the reaction. We are continuing to explore the generality and synthetic application of these acylals and the effect of other Lewis acids on reaction rate and yields. Additional findings will be reported.

\section{Experimental Section}

General procedures. All compounds were identified by comparison of their spectral data and physical properties with those of the authentic samples. ${ }^{1} \mathrm{H}$ NMR and ${ }^{13} \mathrm{C}$ NMR spectra were recorded on a BRUKER DRX-500 AVANCE NMR spectrometer using DMSO-d ${ }_{6}$ as solvent. IR spectra were recorded on Mattson 1000 FT-IR spectrometer using KBr pellets. Zinc chloride, tungstophosphoric and molybdophosphoric acid, ethyl acetoacetate, urea and thiourea were purchased from Merck chemical company. Acylals were prepared according to the procedures reported in the literature. ${ }^{33,34}$

\section{General procedure for PW-catalyzed synthesis of 3, 4-dihydropyrimidin-2(1H)-ones and thiones}

A mixture of an acylal $1(1 \mathrm{mmol}), \beta$-ketoester $2(1 \mathrm{mmol})$, urea or thiourea $3(1.2 \mathrm{mmol})$, and $\mathrm{H}_{3} \mathrm{PW}_{12} \mathrm{O}_{40}(\mathrm{PW})(0.1 \mathrm{mmol})$ was heated at $100{ }^{\circ} \mathrm{C}$ in an oil bath. After completion of reaction (indicated by TLC), the mixture was dissolved in ethanol and poured into cold water. The resulting precipitate was filtered and recrystallized from ethanol. The catalyst remaining in the aqueous phase recovered by removing the water under reduced pressure.

\section{Spectral data for selected compound}

Compound 4a: white powder. IR (KBr): 1650, 1699, 2982, 3131, $3230 \mathrm{~cm}^{-1} .{ }^{1} \mathrm{H}$ NMR (DMSO$\left.\mathrm{d}_{6}\right): \delta=1.10\left(\mathrm{t}, \mathrm{J}=7.1 \mathrm{~Hz}, 3 \mathrm{H}, \mathrm{CH}_{3}\right), 2.25\left(\mathrm{~s}, 3 \mathrm{H}, \mathrm{CH}_{3}\right), 3.99\left(\mathrm{q}, \mathrm{J}=7.1 \mathrm{~Hz}, 2 \mathrm{H}, \mathrm{CH}_{2}\right), 5.15(\mathrm{~d}, \mathrm{~J}$ $=3.2 \mathrm{~Hz}, 1 \mathrm{H}, \mathrm{CH}), 7.23-7.34(\mathrm{~m}, 5 \mathrm{H}$, arom $\mathrm{CH}), 7.73(\mathrm{~s}, 1 \mathrm{H}, \mathrm{NH}), 9.18(\mathrm{~s}, 1 \mathrm{H}, \mathrm{NH}) \cdot{ }^{13} \mathrm{C} \mathrm{NMR}$ $\left(\right.$ DMSO-d $\left._{6}\right) \delta=14.94,18.64,54.83,60.04,100.13,127.11,128.12,129.25,145.73,149.22$, $152.99,166.20$. 


\section{Acknowledgements}

Support of this research by the Faculty of Research of Shahid Bahonar University is gratefully acknowledged.

\section{References and Notes}

1. Clark, J. H. Green Chem. 1999, 1, 1.

2. Das, J.; Parida, K. M. J. Mol. Catal. A: Chem. 2007, 264, 248.

3. Qian, Q.; Zhang, S.; Yuan, G. Catal. Commun. 2007, 8, 483.

4. Da Silva Rocha, K. A.; Robles-Dutenhefner, P. A.; Sousa, E. M. B.; Kozhevnikova, E. F.; Kozhevnikov, I. V.; Gusevskaya, E. V. Appl. Catal. A 2007, 317, 171.

5. Armstrong, R. W.; Combs, A. P.; Tempest, P. A.; Brown, S. D.; Keating, T. A. Acc. Chem. Res. 1996, 29, 123.

6. Dax, S. L.; McNally, J. J.; Youngman, M. A. Curr. Med. Chem. 1999, 6, 255.

7. Rovnyak, G. C.; Kimball, S. D.; Beyer, B.; Cucinotta, G.; Dimarco, J. D.; Gougoutas, J.; Hedberg, A.; Malley, M.; MaCarthy, J. P.; Zhang, R.; Mereland, S. J. Med. Chem. 1995, 38, 119.

8. Kappe, C. O.; Fabian, W. M. F.; Semones, M. A. Tetrahedron 1997, 53, 2803.

9. Atwal, K. S.; Swanson, B. N.; Unger, S. E.; Floyd, D. M.; Moreland, S.; Hedberg, A.; O’Reilly, B. C. J. Med. Chem. 1991, 34, 806.

10. Biginelli, P. Gazz. Chim. Ital. 1893, 23, 360.

11. Chari, M. A.; Shobha, D.; Kumar, T. K.; Dubey, P. K. ARKIVOC 2005, (xv), 74.

12. Fu, N. Y.; Yuan, Y. F.; Cao, Z.; Wang, S. W.; Wang, J. T.; Peppe, C. Tetrahedron 2002, 58, 4801.

13. Su, W.; Li, J.; Zhengb, Z.; Shen, Y. Tetrahedron Lett. 2005, 46, 6037.

14. Desai, B.; Dallinger, D.; Kappe, C. O. Tetrahedron 2006, 62, 4651.

15. Gholap, A. R.; Venkatesan, K.; Daniel, T.; Lahoti, R. J.; Srinivasan, K. V. Green Chem. 2004, 6,147.

16. Kappe, C. O. Acc. Chem. Res. 2000, 33, 879.

17. Nilson, B. L.; Overman, L. E. J. Org. Chem. 2006, 71, 7706.

18. Kochhar, K. S.; Bal, B. S.; Deshpande, R. P.; Rajadhyaksha, S. N.; Pinnick, H. W. J. Org. Chem. 1983, 48, 1765.

19. Sandberg, M.; Sydnes, L. K. Org. Lett. 2000, 2, 687.

20. Trost, B. M.; Lee, C. J. Am. Chem. Soc. 2001, 123, 12191.

21. Trost, B. M.; Lee, C. B.; Weiss, J. M. J. Am. Chem. Soc. 1995, 117, 7247.

22. Banks, R. E.; Miller, J. A.; Nunn, M. J.; Stanley, P.; Weakley, T. J. R.; Ullah, Z. J. Chem. Soc., Perkin Trans. 1 1981, 1096.

23. Yu, Y.; Liu, D.; Liu, C.; Luo, G. Bioorg. Med. Chem. Lett. 2007, 17, 3508. 
24. Shaabani, A.; Bazgir, A.; Teimuri, F. Tetrahedron Lett. 2003, 44, 857.

25. Shirini, F.; Marjani, K.; Taherpour Nahzomi, H. ARKIVOC 2007, (i), 51.

26. Bigdeli, M. A.; Jafari, S.; Mahdavinia, G. H.; Hazarkhani, H. Catal. Commun. 2007, 8, 1641.

27. Kumar, A.; Maurya, R. A. Tetrahedron Letters 2007, 48, 4569.

28. Fu, N. Y.; Yuan, Y. F.; Cao, Z.; Wang, S. W.; Wang, J. T.; Peppe, C. Tetrahedron 2002, 58, 4801.

29. Nandurkar, N. S.; Bhanushali, M. J.; Bhor, M. D.; Bhanage, B. M. J. Mol. Catal. A: Chem. 2007, 271, 14.

30. Heravi, M. M.; Derikvand, F.; Bamoharram, F. F. J. Mol. Catal. A: Chem. 2005, 242, 173.

31. Heravi, M. M.; Bakhtiari, K.; Bamoharram, F. F. Catal. Commun. 2006, 7, 373.

32. El Badaoui, H.; Bazi, F.; Tahir, R.; Lazrek, H. B.; Sebti, S. Catal. Commun. 2005, 6, 455.

33. Heravi, M. M.; Bakhtiari, K.; Bamoharram, F. F. Catal. Commun. 2006, 7, 499.

34. Romanelli, G. P.; Thomas, H. J.; Baronettic, G. T.; Autinoa, J. C. Tetrahedron Lett. 2003, 44, 1301. 